\title{
Recomendações para a atuação dos fisioterapeutas nos casos de oxigenação por membrana extracorpórea (ECMO)*
}

\author{
Recommendations for physiotherapy in patients on Extracorporeal Membrane Oxygenation \\ $(\mathrm{ECMO})^{*}$
}
LAGES, Natália Coronel de Lima'; TIMENETSKY, Karina Tavares²; em nome do Comitê COVID-19 da ASSOBRAFIR.

\begin{abstract}
Resumo
A Oxigenação por Membrana Extracorpórea (ECMO) é indicada quando o paciente desenvolve falência respiratória hipoxêmica refratária às estratégias convencionais de resgate, muito comum nos pacientes acometidos pela COVID-19. A manutenção de determinadas condições por 72 horas, tais como a relação da pressão parcial de oxigênio com a fração inspirada de oxigênio $\left(\mathrm{PaO}_{2} / \mathrm{FiO}_{2}\right)<100 \mathrm{mmHg}$, complacência estática $<10 \mathrm{ml} / \mathrm{cmH} 20$ e/ou $\mathrm{pH}<7,2$ com pressão parcial de dióxido de carbono $\left(\mathrm{PaCO}_{2}\right)>60 \mathrm{mmHg}$ tem sido utilizadas, por centros especializados, como critérios para indicação precoce de ECMO nesses pacientes. A ventilação mecânica dos pacientes em ECMO deve sempre objetivar proteção pulmonar e diafragmática e a estratégia ventilatória deve ser individualizada. A mobilização e os exercícios terapêuticos devem ser implementados precocemente nestes pacientes, sendo considerados procedimentos seguros quando respeitadas as recomendações. A indicação de fisioterapia deve levar em consideração os ajustes da ECMO no momento da intervenção.
\end{abstract}

Palavras-chave: Fisioterapia; Ventilação Mecânica; ECMO; COVID-19.

* Revisado por membros do Comitê COVID-19 da ASSOBRAFIR, nomeado por meio do memorando Nº 003/2020. Esta publicação é uma atualização da Comunicação oficial "Recomendações para a atuação dos fisioterapeutas nos casos de oxigenação por membrana extracorpórea (ECMO)", chancelada pelo Comitê COVID-19 da ASSOBRAFIR, originalmente escrita pelos mesmos autores e divulgada em 23/04/2020 no endereço eletrônico https://assobrafir.com. br/covid-19 ecmo/.

1 Hospital Universitário Pedro Ernesto (HUPE), Serviço de Cirurgia Cardíaca, Universidade do Estado do Rio de Janeiro (UERJ), Rio de Janeiro, Rio de Janeiro, Brasil. AmericanCor Hospital (ACH), Rio de Janeiro, Rio de Janeiro, Brasil. Programa de Pós-Graduação em Terapia Intensiva do Centro Universitário Augusto Motta (UNISUAM), Rio de Janeiro, Rio de Janeiro, Brasil. Email: nataliacoronellages@gmail.com. NCL -https://orcid.org/0000-0001-5121-9123

2 Departamento de Pacientes Graves do Hospital Israelita Albert Einstein, São Paulo, SP, Brasil. KTT -https://orcid.org/0000-0002-4176-2445 


\section{Abstract}

Extracorporeal Membrane Oxygenation (ECMO) is indicated for acute hypoxemic respiratory failure refractory to conventional rescue therapies, a condition commonly observed in COVID-19 patients. Criteria such as a partial pressure of oxygen to inspired oxygen fraction ratio $\left(\mathrm{PaO}_{2} / \mathrm{FiO}_{2}\right)<100 \mathrm{mmHg}$, static lung compliance $<10 \mathrm{ml} / \mathrm{cmH} 20$ and/or $\mathrm{pH}<7.2$ with a partial pressure of carbon dioxide $\left(\mathrm{PaCO}_{2}\right)>60 \mathrm{mmHg}$ for at least 72 hours, have been used by some specialists for early indication of ECMO in these patients. Mechanical ventilation during ECMO must be individualized and should focus on lung and diaphragmatic protection. Mobilization and therapeutic exercises should be implemented early in these patients and both are considered safe procedures, if safety recommendations are followed. Physiotherapy prescription should take into account the ECMO settings at the time of the intervention.

Keywords: Physiotherapy; Mechanical Ventilation; ECMO; COVID-19.

\section{Objetivo}

A Oxigenação por Membrana Extracorpórea (ECMO) é um recurso disponível em alguns centros do Brasil e pode ser benéfico no tratamento de pacientes com hipoxemia refratária difícil de ser corrigida pela ventilação pulmonar, como nos casos da COVID-19. Neste documento, a ASSOBRAFIR posiciona-se em relação à indicação da ECMO e à atuação dos fisioterapeutas durante o seu uso em pacientes com COVID-19.

\section{Introdução}

A ECMO é uma modalidade de suporte de vida extracorpóreo que possibilita suporte temporário à falência da função pulmonar e/ou cardíaca, refratária ao tratamento clínico convencional (ventilação mecânica invasiva, estratégia protetora e/ou ventilação prona). O primeiro relato do uso com sucesso de um dispositivo de assistência circulatória extracorpórea data de 1954, durante uma cirurgia cardíaca e, apenas em 1972, foi utilizado no contexto de falência ventilatória na terapia intensiva. O primeiro estudo multicêntrico e randomizado que avaliou o emprego da ECMO no contexto de insuficiência respiratória foi publicado em 1979. Desde as primeiras descrições desta técnica, melhorias significativas ocorreram no dispositivo, no manejo e, consequentemente, nos desfechos dos pacientes em $\mathrm{ECMO}^{1}$.

Diversos estudos descrevem a utilização de ECMO em pacientes com choque cardiogênico refratário, síndrome do desconforto respiratório agudo (SDRA) refratário ao suporte clínico convencional, ou ainda em parada cardiorrespiratória, sendo as indicações de instalação e manuseio descritas nas Orientações da ELSO (Extracorporeal Life Support Organization) para insuficiência cardíaca e respiratória do adulto (www.elso.org) ${ }^{1,2}$. A ECMO é indicada para pacientes com alto risco de morte e, quando se trata de SDRA, existem vários métodos para estimar este risco, todos incluindo a relação $\mathrm{PaO}_{2} / \mathrm{FiO}_{2}$ (relação entre pressão parcial de oxigênio no sangue arterial e fração inspirada de oxigênio) abaixo de 100, apesar da terapia otimizada ${ }^{3}$. Muitos algoritmos para o tratamento da SDRA foram adaptados e podem ser úteis para a decisão clínica, como o apresentado na Figura $1^{3}$. 
Figura 1: Algoritmo para manejo da síndrome do desconforto respiratório agudo.

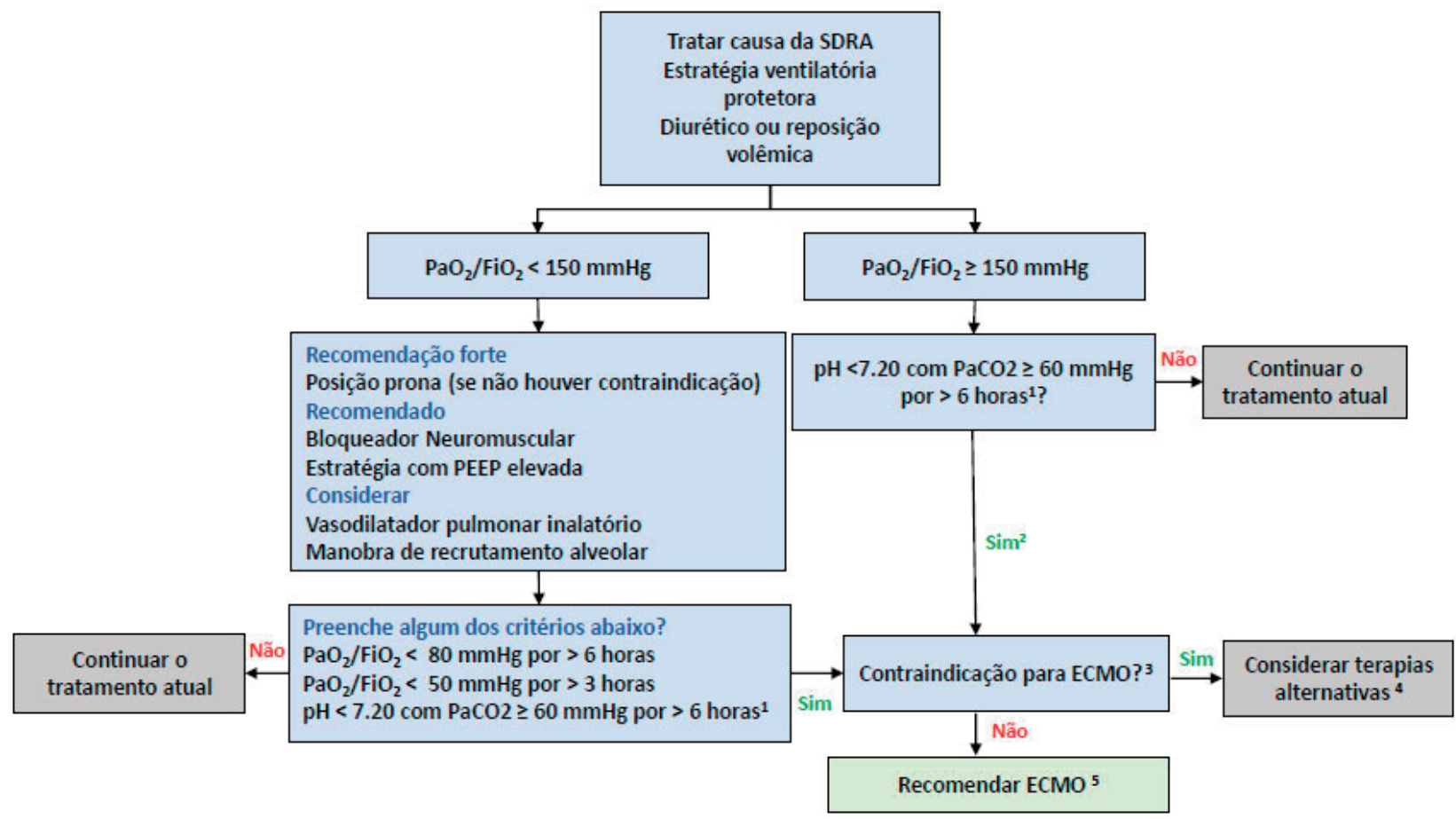

SDRA = síndrome do desconforto respiratório agudo; $\mathrm{PaO}_{2} / \mathrm{FiO}_{2}=$ relação entre pressão parcial de oxigênio no sangue arterial e fração inspirada de oxigênio; PEEP = pressão positiva no final da expiração; ECMO = oxigenação por membrana extracorpórea; $\mathrm{PaCO}_{2}=$ pressão parcial do dióxido de carbono no sangue arterial. ${ }^{1}$ Considerar aumento na frequência respiratória para até 35 incursões por minuto (ipm) e parâmetros ventilatórios ajustados para manter uma pressão de platô $\leqslant 30 \mathrm{cmH} 2 \mathrm{O} .{ }^{2}$ Considerar bloqueio neuromuscular. ${ }^{3}$ Não existe consenso quanto a contraindicação absoluta, exceto doença pulmonar terminal sem possibilidade de transplante pulmonar; critério de exclusão utilizado no estudo EOLIA ${ }^{4}$ pode ser uma abordagem conservadora nas contraindicações de ECMO. ${ }^{4}$ Exemplos: bloqueio neuromuscular, estratégia com PEEP elevada, vasodilatador pulmonar inalatório, manobra de recrutamento alveolar. ${ }^{5}$ Recomendação precoce de ECMO baseada nos critérios do estudo EOLIA.

Fonte: Adaptado de ELSO Guidance Document: Use of ECMO in Covid-19 patients during the pandemic.. ${ }^{3}$

Nos pacientes com indicação de ECMO, seu início deve ser imediato, desde que realizado em um centro experiente. A ECMO como forma de resgate, que envolveria postergar a ECMO até a descompensação, pode ser um caminho no atual cenário de crise, entretanto, não é uma alternativa suportada pelas evidências disponíveis ${ }^{3,4}$.

A ECMO é indicada quando o paciente desenvolve falência respiratória hipoxêmica refratária às estratégias convencionais de resgate como a titulação de PEEP e a posição prona, o que em muitas vezes acontece com os pacientes acometidos pela COVID-19. A manutenção de determinadas condições por 72 horas, tais como $\mathrm{PaO}_{2} / \mathrm{FiO}_{2}<100 \mathrm{mmHg}$, complacência estática (Cest) $<10 \mathrm{ml} /$ $\mathrm{cmH} 20$ e/ou pH $<7,2$ com $\mathrm{PaCO}_{2}>60 \mathrm{mmHg}$ tem sido utilizadas, por centros especializados, como critérios para indicação precoce de ECMO nesses pacientes ${ }^{1,5}$.

No contexto da COVID-19, apresentamos o Quadro 1 com base nos critérios utilizados pelo estudo EOLIA4 e sugeridos pela $\mathrm{ELSO}^{3}$, com as condições a serem avaliadas na indicação de ECMO. É importante ressaltar que tais condições não representam indicações absolutas e devem ser ajustadas 
de acordo com a capacidade de cada serviço. A elegibilidade de pacientes para ECMO pode ser individualizada à critério dos centros especializados e vale destacar que não existe consenso quanto a contraindicação absoluta, exceto doença pulmonar terminal sem possibilidade de transplante pulmonar. A ELSO não recomenda ECMO em parada cardiorrespiratória durante a pandemia por COVID-19 $9^{1-3}$.

Quadro1 | Indicações da ECMO para pacientes com COVID-19,3,3,4.

\begin{tabular}{|l|}
\hline INDICAÇõES DE ECMO \\
\hline Idade $<65$ anos \\
$\mathrm{VMI}<7$ dias \\
$\mathrm{Pplatô}<30 \mathrm{cmH}_{2} \mathrm{O}$ \\
$\mathrm{PaO}_{2} / \mathrm{FiO}_{2}<50 \mathrm{mmHg}$ por $3 \mathrm{~h}$ ou \\
$\mathrm{PaO}_{2} / \mathrm{FiO}_{2}<80 \mathrm{mmHg}$ por $6 \mathrm{~h}$ \\
$\mathrm{pH}<7,20$ com PaCO${ }_{2}>60 \mathrm{mmHg}$ por $6 \mathrm{~h}$ \\
$\mathrm{VMI}=$ ventilação mecânica invasiva; Pplatô = pressão de platô; $\mathrm{PaO}_{2}$ \\
$=$ pressão arterial de oxigênio; FiO ${ }_{2}=$ fração inspirada de oxigênio; \\
$\mathrm{PaO} / / \mathrm{FiO}_{2}=$ relação da pressão arterial de oxigênio e fração inspirada \\
de oxigênio; $\mathrm{PaCO}_{2}=$ pressão arterial de dióxido de carbono.
\end{tabular}

\section{Configurações e componentes do circuito de ECMO} paciente ${ }^{2}$ :

O circuito de ECMO será configurado de acordo com o tipo de suporte indicado para o Suporte veno-arterial (V/A):

- Indicação primária para falência circulatória.

- Configuração necessária para suporte circulatório, além de ser apropriada para suporte respiratório.

\section{Suporte veno-venoso (V/V):}

- Indicação primária para falência pulmonar aguda.

- Apenas para suporte ventilatório.

- Não proporciona suporte circulatório.

Suporte artério-venoso $(\mathrm{A} / \mathrm{V})$ :

- Indicação primária exclusiva para remoção de $\mathrm{CO}_{2}$.

- Dispositivo limitado a um baixo fluxo sanguíneo.

- Utilizado apenas para remoção de $\mathrm{CO}_{2}$.

Os pacientes com COVID-19 podem apresentar complicações pulmonares evoluindo para um quadro de hipoxemia grave. Na presença de um quadro hipoxêmico refratário ao suporte clínico convencional, a modalidade de primeira escolha é a veno-venosa, podendo ser convertida em venoarterial caso o paciente apresente falência cardíaca secundária. 
Após a canulação para ECMO, o paciente deve estar sedado e sob bloqueio neuromuscular para ajustes iniciais de parâmetros ventilatórios e titulação de PEEP. Não é recomendado permitir ventilação espontânea nas primeiras 48 horas de ECMO. Além disso, primeiramente deverá ser reduzida a $\mathrm{FiO}_{2}$ do ventilador mecânico e, posteriormente, a da membrana, para manutenção da $\mathrm{SpO}_{2}$ (saturação periférica de oxigênio) entre 90 e $96 \%^{4-7}$. Especialistas sugerem, no contexto da COVID-19, que a $\mathrm{SpO}_{2}$ deva ser mantida entre 92 e 96\% pela presença de fenômenos micro trombóticos associados.

Membrana ou oxigenador é a porção do circuito onde ocorre a oxigenação do sangue e remoção de $\mathrm{CO}_{2}$. Dependendo do modelo de membrana empregado, a taxa de fluxo (capacidade de troca gasosa ou capacidade máxima de entrega de oxigênio) difere de acordo com suas propriedades (área de superfície e o material utilizado). A capacidade de troca gasosa da membrana deve ser considerada no momento da sua escolha. A fração ofertada de oxigênio é ajustada por meio de um botão localizado no misturador de gás do equipamento de $\mathrm{ECMO}^{2}$. Um esquema conceitual do sistema de oxigenação por membrana extracorpórea está representado nas Figuras 2A e 2B.

Figura 2A | Esquema conceitual do sistema de oxigenação por membrana extracorpórea (ECMO).

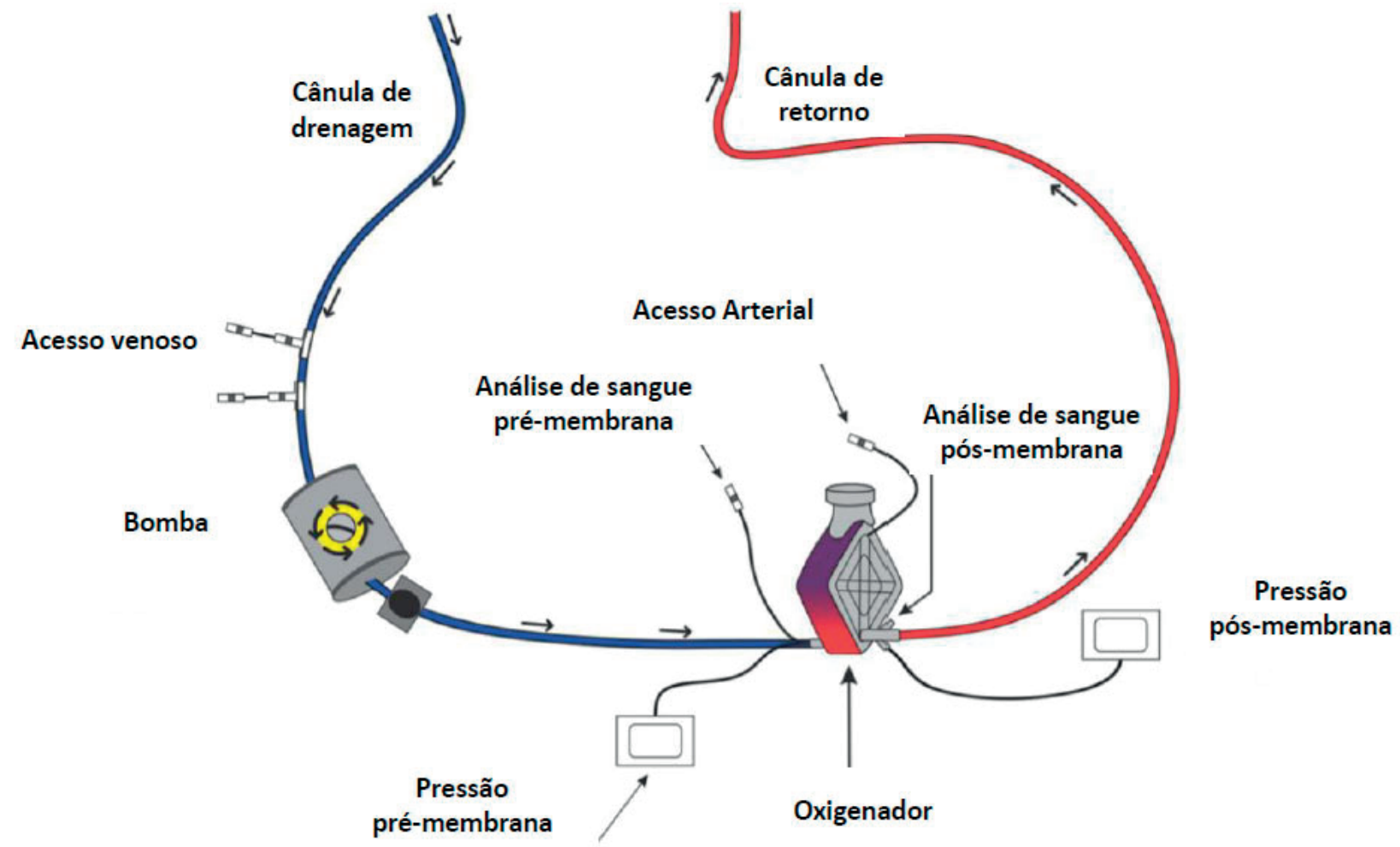

Fonte: Adaptado de Chaves e cols., $2019^{1}$. 
Figura 2B | Esquema conceitual do oxigenador do sistema de oxigenação por membrana extracorpórea (ECMO).

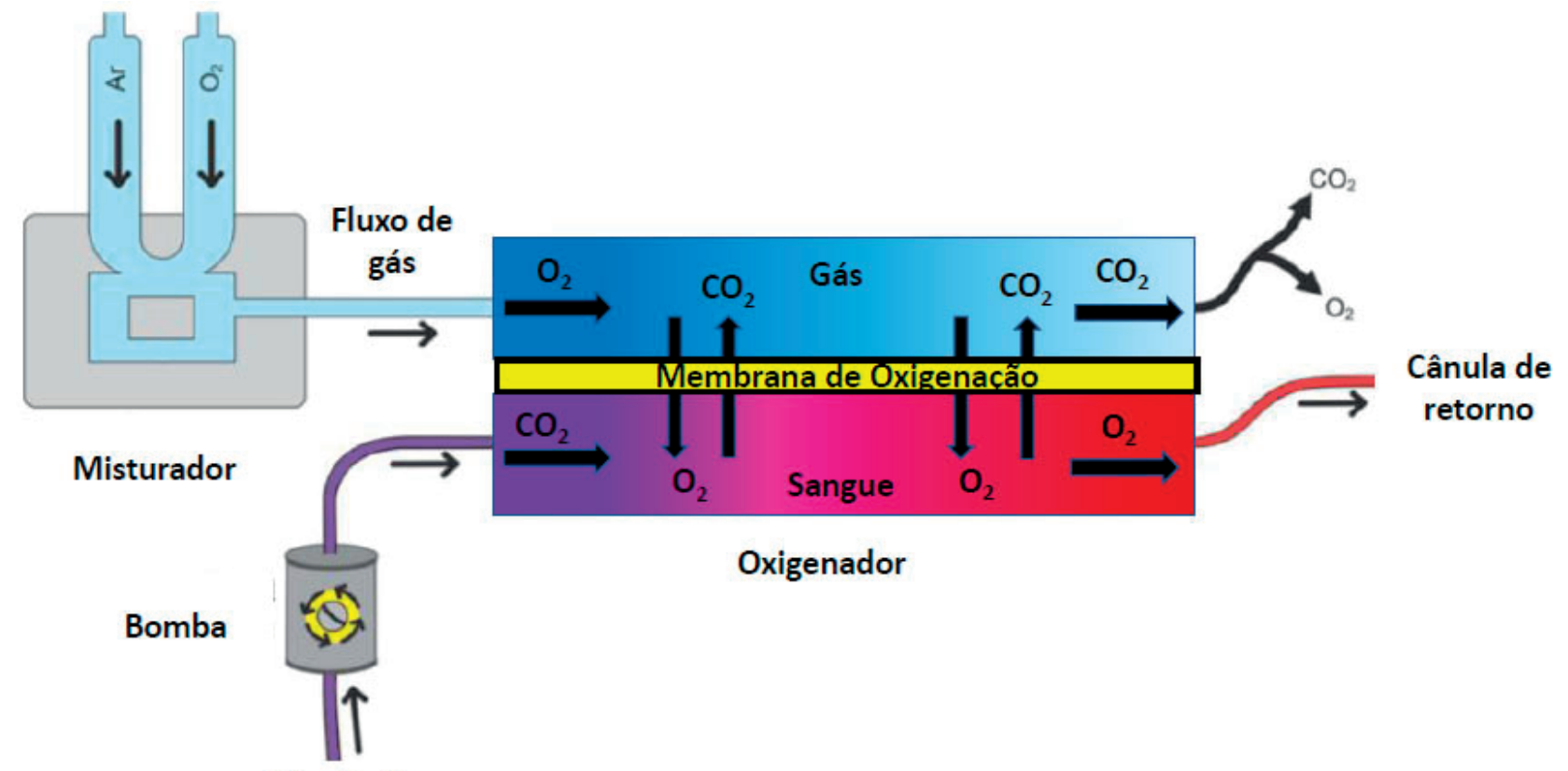

Cânula de drenagem

Fonte: Adaptado de Chaves e cols., 2019 '.

No circuito da ECMO, o fluxo de gás para remoção de $\mathrm{CO}_{2}$ é ofertado por meio de um fluxômetro e, inicialmente, os fluxos de gás e de sangue devem guardar a relação de 1:1 nos casos de suporte pleno com ECMO de alto fluxo sanguíneo. O fluxo de gás deverá ser ajustado de acordo com o resultado da gasometria arterial para manutenção de $\mathrm{PaCO}_{2}$ dentro dos limites desejáveis (35 e 45 $\mathrm{mmHg}$ ). A transferência de $\mathrm{CO}_{2}$ através da membrana ocorre em velocidade superior à transferência de oxigênio, logo, é necessário atentar para que o $\mathrm{CO}_{2}$ não seja removido em excesso ou muito rapidamente. A rápida remoção de $\mathrm{CO}_{2}$ do sangue pode culminar em vasoconstrição e hipoperfusão cerebral. A remoção de $\mathrm{CO}_{2}$ do sangue não deve exceder $10 \mathrm{mmHg}$ por hora ${ }^{1,2}$. O aumento do fluxo de gás aumenta o clearance de $\mathrm{CO}_{2}$ (velocidade de remoção do sangue), porém, sem melhora da oxigenação. $\mathrm{O}$ incremento da oxigenação ocorre por meio do aumento da fração de oxigênio ofertada e/ou por meio do aumento do fluxo de sangue através da membrana do oxigenador ${ }^{1,2}$. É preciso atentar para condensação de vapor d'água na membrana, o que resulta em redução significativa do clearance de $\mathrm{CO}_{2}^{2}$.

O manejo adequado da VMI (ventilação mecânica invasiva) é fundamental para proteção pulmonar e pode promover um benefício adicional ao paciente durante a ECMO. Para pacientes com falência cardíaca severa em ECMO V/A, o fluxo através da artéria pulmonar pode estar muito reduzido e a manutenção de uma ventilação alveolar normal pode ocasionar hiperventilação pulmonar, resultando em alcalose tecidual, acelerando o processo de trombose vascular pulmonar na presença de grave lesão pulmonar.

A pressão positiva nas vias aéreas também afeta a pré-carga e a pós-carga tanto do ventrículo direito (VD), como do ventrículo esquerdo (VE). Os pacientes em ECMO V/A com falência de 
VD podem sofrer efeitos negativos de altos níveis de pressão positiva ao final da expiração (PEEP), enquanto pacientes com falência de VE podem se beneficiar de níveis elevados de PEEP, reduzindo a probabilidade de edema pulmonar ${ }^{9-12}$.

Uma estratégia ventilatória ultra protetora pode ser empregada em pacientes em uso de ECMO, uma vez que a membrana do dispositivo é capaz de manter as taxas de oxigênio e gás carbônico dentro de valores desejados. Volumes pulmonares reduzidos são recomendados, entretanto, aumentam as áreas de atelectasia, gerando maior desequilíbrio da relação ventilação/perfusão. Desta forma, conclui-se que níveis elevados de PEEP geralmente são recomendados, entretanto, o paciente deve ser avaliado de forma individualizada e suas limitações cardíacas e hemodinâmicas devem ser consideradas nesse ajuste ${ }^{7}$.

\section{Manejo ventilatório do paciente COVID-19 em ECMO}

ECMO permite ventilação mecânica protetora, além de proteção diafragmática, possibilitando a ventilação espontânea precoce com um esforço inspiratório dentro dos limites fisiológicos aceitáveis ${ }^{6}$. O manejo inadequado da ventilação mecânica pode proporcionar ainda mais danos aos pulmões (injúria pulmonar induzida pelo ventilador, do inglês ventilator-induced lung injury - VILI) e o esforço muscular inadequado, seja ele insuficiente (P0.1 - pressão inspiratória gerada em $100 \mathrm{~ms}$ $\left.<2,0 \mathrm{cmH}_{2} \mathrm{O}\right)$ ou excessivo $\left(\mathrm{P} 0.1>4,0 \mathrm{cmH}_{2} \mathrm{O}\right)$, pode levar à atrofia diafragmática ou miotrauma, respectivamente ${ }^{6}$. A monitorização do drive ventilatório e do esforço do paciente são fundamentais para minimização da P-SILI (do inglês patient self inflected lung injury, lesão pulmonar auto-infligida pelo paciente) durante a respiração espontânea. O suporte ventilatório em ECMO é ainda um tópico em debate e diversas estratégias ultra protetoras vem sendo propostas para reduzir a VILI, dentre elas, a minimização de pressão de platô (Pplat), pressão de distensão ou "driving pressure" (DP), poder mecânico (do inglês mechanical power - MP), frequência respiratória (FR) e volume corrente $\left(\mathrm{V}_{\mathrm{T}}\right)$. Como consenso, temos apenas a garantia de troca gasosa adequada com menor MP possível'.

Após a canulação para ECMO, o paciente deve estar sedado e curarizado para ajustes iniciais de parâmetros ventilatórios e titulação de PEEP, como demonstrado no Quadro 2. O uso contínuo de bloqueador neuromuscular não está indicado, devendo, este, ser administrado a critério médico. A ventilação pulmonar ultra protetora em ECMO geralmente requer PEEP mais elevada, entretanto, esta PEEP deve ser suficiente para sustentar o recrutamento alveolar sem causar hiperdistensão. Sua titulação pode ser feita por meio da DP. Em 2017, demonstrou-se diferentes níveis ótimos de PEEP nos pacientes com SDRA em ECMO avaliados pela tomografia de impedância elétrica, levantando a necessidade de individualização do ajuste de PEEP desses pacientes ${ }^{13}$. Não se deve permitir ventilação espontânea nas primeiras $48 \mathrm{~h}$ de ECMO, tendo em vista que o esforço muscular respiratório maior e descontrolado poderia dificultar o manejo ventilatório e o desmame dos pacientes mais graves. Durante a permanência dos pacientes em ventilação mecânica controlada é possível manter a relação entre o tempo inspiratório e o tempo expiratório (I:E) em 2:1, no entanto, à medida que o paciente torna-se mais estável e apresenta melhora hemodinâmica, os níveis de sedação são reduzidos. Neste momento, a relação I:E deve manter uma proporção mais próxima da fisiológica, aproximadamente de 1:2 1,6.

Após a estabilização do paciente, deve-se realizar a coleta de gasometria arterial (pósmembrana) e gasometria venosa (pré-membrana) para ajustes ventilatórios e circulatórios. Os ajustes circulatórios são realizados por profissional especialista, perfusionista, médico intensivista ou cirurgião cardíaco. O manejo ventilatório e dos gases da ECMO deve ser conduzido por profissional especialista em ECMO, fisioterapeuta ou médico intensivista com experiência em ventilação 
mecânica. As decisões são multidisciplinares e a estratégia ventilatória é complementar e não pode ser dissociada da estratégia de oxigenação e remoção de $\mathrm{CO}_{2}$ através da membrana. Assim, as ações precisam ser planejadas, executadas e monitoradas de forma simultânea. Por exemplo, se o fluxo de sangue desviado para o dispositivo for reduzido, haverá limitação da troca gasosa pela redução da vazão de sangue a ser arterializado e, neste momento, a estratégia ventilatória deve retornar gradativamente à parâmetros mais fisiológicos ${ }^{2}$.

Quadro 2 | Sugestão de parâmetros ventilatórios iniciais para pacientes em ECMO ${ }^{1,6,7,15,16}$.

\begin{tabular}{|l|}
\hline PARÂMETROS VENTILATÓRIO INICIAIS \\
\hline Volume corrente $=3$ a $4 \mathrm{ml} / \mathrm{Kg}$ de peso predito \\
Pressão de platô $\leq 28 \mathrm{cmH}_{2} \mathrm{O}$ \\
Driving Pressure (DP) $\leq 10 \mathrm{cmH}_{2} \mathrm{O}$ \\
Frequência respiratória $=5-10 \mathrm{irpm}$ \\
$\mathrm{FiO}_{2}$ na membrana de $\mathrm{ECMO}=100 \%$ \\
$\mathrm{FiO}_{2}$ no ventilador mecânico $=50 \%$ \\
$\mathrm{PEEP}=10-15 \mathrm{cmH}_{2} 0$ ou titulação pela menor DP \\
Relação I:E $=2: 1$ nas primeiras $24 \mathrm{~h}-48 \mathrm{~h}$ \\
(*durante ciclo controlado apenas)
\end{tabular}

Driving Pressure (delta de pressão); $\mathrm{FiO}_{2}=$ fração inspirada de oxigênio

Quadro 3 | Sugestão para monitorização ventilatória de pacientes com COVID-19 na modalidade ventilação mecânica (VM) controlada e na modalidade VM espontânea ${ }^{1,6,714-16}$.

\begin{tabular}{|ll|}
\hline VENTILAÇÃO CONTROLADA & VENTILAÇÃO ESPONTÂNEA \\
\hline Volume corrente $(\mathrm{VC})=3$ a $4 \mathrm{ml} / \mathrm{Kg}$ de peso predito & $\mathrm{VC}=4$ a $6 \mathrm{ml} / \mathrm{Kg}$ de peso predito \\
$\mathrm{FiO}_{2}$ menor possível para $\mathrm{SpO}_{2}$ entre $92 \%$ e $96 \%$ ou para $\mathrm{PaO}_{2}$ desejada \\
Pressão de platô $\leq 28 \mathrm{cmH}_{2} \mathrm{O}$ & Pressão de pico $<25 \mathrm{cmH}_{2} \mathrm{O}$ \\
Driving Pressure (DP) $\leq 10 \mathrm{cmH}_{2} \mathrm{O}$ & DP Transpulmonar $=10$ a $12 \mathrm{cmH}_{2} \mathrm{O}$ \\
--- & DP Esofágico $=4$ a $8 \mathrm{cmH}_{2} \mathrm{O}$ \\
Frequência respiratória $=5$ a 10 irpm & Frequência respiratória $\leq 25$ irpm \\
PEEP 10 a 15 e/ou titulada para menor DP & PEEP 10 a 15 e/ou titulada para menor DP \\
Relação I:E $=1: 2$ & --- \\
--- & PO.1 $=2,0$ a $4,0 \mathrm{cmH}_{2} \mathrm{O}$ \\
\hline
\end{tabular}

$\mathrm{FiO}_{2}=$ fração inspirada de oxigênio; $\mathrm{SpO}_{2}=$ saturação periférica de oxigênio; $\mathrm{PaO}_{2}=$ pressão arterial de oxigênio; P0.1 = pressão inspiratória gerada em $100 \mathrm{~ms}$. 


\section{Mobilização e reabilitação de pacientes com COVID-19 em ECMO}

A colocação do paciente em ECMO não é contraindicação para a realização de fisioterapia, pelo contrário. A mobilização e os exercícios terapêuticos devem ser implementados precocemente em pacientes em uso de ECMO, sendo considerados procedimentos seguros quando respeitadas as recomendações. Pacientes submetidos a reabilitação precoce apresentam maior chance de retorno às atividades de vida diária prévias com independência funcional, menor incidência de delirium, redução do tempo em VMI, assim como menor tempo de permanência hospitalar e em unidades de terapia intensiva ${ }^{17}$.

Nos últimos anos, a incidência de pacientes despertos em ECMO aumentou significativamente, reduzindo assim os efeitos negativos associados à sedação ${ }^{18}$. Essa mudança no perfil dos pacientes em ECMO chamou atenção para necessidade de novos estudos na área da reabilitação funcional precoce. Ainda não há diretrizes sobre como essa intervenção deva ser conduzida pelos fisioterapeutas neste grupo específico de pacientes, porém, alguns centros especializados publicaram rotinas seguras e de boas práticas ${ }^{19}$. No Brasil, essa abordagem ainda é recente e com grandes desafios na prática clínica. Contudo, é possível e seguro realizá-la com a formação e treinamento de equipes especializadas.

O programa de reabilitação precisa estar adequado ao momento clínico do paciente. Além disso, a equipe multidisciplinar deve ser satisfatoriamente dimensionada ao nível de atendimento. $\hat{E}$ necessário considerar aspectos como a o estágio da recuperação do paciente, o sítio de canulação, $o$ nível de sedação e de anticoagulação, além de observar os efeitos dos procedimentos fisioterapêuticos empregados sobre o fluxo da $\mathrm{ECMO}^{19}$.

A reabilitação motora e funcional durante a ECMO requer cuidado e uma equipe multidisciplinar envolvida no planejamento, desenvolvimento e acompanhamento do processo. A composição desta equipe pode variar de acordo com a instituição, porém, é basicamente composta por: médico/a especialista em ECMO (cirurgião ou intensivista), enfermeiro/a especialista em ECMO, enfermeiro/a intensivista, perfusionista, fisioterapeuta intensivista e fisioterapeuta com experiência em $\mathrm{ECMO}^{19}$.

Pacientes em ECMO têm cânulas calibrosas em vasos centrais (femoral ou jugular) para permitir um alto fluxo contínuo no circuito da ECMO. Qualquer distúrbio neste fluxo, por posicionamento inadequado do paciente ou por deslocamento da cânula, pode gerar hipoxemia grave ${ }^{18}$. A possibilidade de complicações graves envolvendo a fisioterapia durante a ECMO reforça a necessidade de práticas seguras validadas, entretanto, não existem protocolos estabelecidos para mobilização e reabilitação de pacientes em ECMO, e sim recomendações, como intervir progressivamente, respeitando os limites e habilidades de cada paciente. A segurança do processo de mobilização e reabilitação pode ser garantida por meio de checklists antes do atendimento. 
Quadro 4 | Sugestão de checklist de segurança para mobilização e reabilitação precoce do paciente COVID-19 em ECMO ${ }^{18,19}$.

\section{CHECKLIST DE SEGURANÇA PARA MOBILIZAÇÃO E REABILITAÇÃO}

- Definir o plano terapêutico para o atendimento

- Definir o quantitativo multiprofissional adequado ao nível de atendimento

- Discutir o nível de monitorização com a equipe de reabilitação

- Avaliar se as doses de inotrópicos e vasopressores são adequadas ao nível de atendimento

- Checar se a anticoagulação está mantida dentro dos limites desejáveis estipulados

- Confirmar por radiografia o posicionamento interno adequado das cânulas

- Conferir as fixações das cânulas e dos componentes do circuito da ECMO

○ Conferir a fixação e realizar a monitorização da via aérea do paciente

\section{Monitorização e segurança do paciente durante a mobilização e reabilitação}

Durante a intervenção fisioterápica do paciente em ECMO recomenda-se a presença de equipe multidisciplinar e se possível dois fisioterapeutas com experiência no processo. Um médico especialista em ECMO, um enfermeiro especialista e outro perfusionista devem estar presentes para garantir a monitorização do fluxo e do circuito da ECMO durante o atendimento. Os sinais vitais devem ser observados e documentados antes, durante e depois do atendimento e qualquer alteração na pressão arterial (PA), frequência cardíaca $(\mathrm{FC})$, saturação periférica de oxigênio $\left(\mathrm{SpO}_{2}\right)$ ou fluxo sanguíneo através das cânulas ou sistema da ECMO devem ser observados. Pacientes com baixa função pulmonar ou que não respondam com incremento da $\mathrm{PaO}_{2}$ após aumento da fração de $\mathrm{O}_{2}$ ofertada pelo oxigenador podem necessitar de aumento no fluxo sanguíneo por meio da ECMO durante o exercício.

A indicação da fisioterapia nos pacientes em ECMO também deve levar em consideração os ajustes da ECMO no momento da intervenção. Caso o fluxo de sangue, $\mathrm{FiO}_{2}$ e fluxo de gás estiverem ajustados em seus valores máximos não seria o momento ideal para realizar a reabilitação, pois caso o paciente apresente piora clínica não será possível realizar ajustes na ECMO.

A equipe de fisioterapia, durante o atendimento, deve estar ciente dos riscos de sangramento nos sítios de canulação, movimentação das cânulas e redução do fluxo sanguíneo durante mudanças no posicionamento do paciente. Se o paciente se queixar de dor, a possibilidade de deslocamento da cânula deve ser investigada. Qualquer membro da equipe pode interromper a progressão da intervenção caso note alteração dos parâmetros de monitorização e, se não for possível retornar o paciente para o leito em caso de um evento adverso, este deve ser posicionado deitado no chão para que as medidas de emergência sejam tomadas.

A reabilitação deve ser progressiva, a evolução do paciente monitorada continuamente e o plano terapêutico ajustado de forma individualizada e diariamente. Caso haja períodos de instabilidade durante a intervenção fisioterápica pode ser necessário rever o planejamento por algumas sessões 
ou retornar ao estágio anterior da programação. As técnicas utilizadas, número de repetições e carga empregada ao exercício devem ser prescritas e conduzidas de forma individualizada, como em qualquer paciente de terapia intensiva, respeitando suas limitações, estágio da reabilitação e checklist de segurança. A progressão das posturas de sentado no leito até a deambulação, passando por sedestação à beira leito, transferência para posição ortostática, sedestação na poltrona, exercícios em posição ortostática, marcha estacionária e deambulação devem ser respeitadas ${ }^{19-21}$.

\section{Considerações Finais}

A ECMO não é uma terapia de primeira opção, entretanto pode ser considerada para pacientes com COVID-19 que não respondem adequadamente aos tratamentos clínicos convencionais de correção da hipoxemia. A decisão de utilização é de responsabilidade da equipe multiprofissional de cada instituição e deve ser avaliada caso a caso com base na capacidade das UTIs, no número de pacientes, na equipe e em outras restrições de recursos. Se o hospital precisar comprometer todos seus recursos com outros pacientes, a ECMO pode não ser considerada pois poderá esgotar os recursos disponíveis, especialmente nesta pandemia de COVID-19. Pacientes mais jovens, sem ou com poucas comorbidades, são prioridade, quando os recursos são limitados. Os profissionais de saúde são considerados de alta prioridade e deve-se reconhecer que essa é uma priorização dinâmica. À medida que os recursos mudam, as prioridades devem mudar com base no que pode ser feito com segurança no ambiente específico do hospital ${ }^{3}$.

A ventilação mecânica dos pacientes em ECMO deve sempre objetivar proteção pulmonar e diafragmática por meio do controle do drive ventilatório e do esforço inspiratório e deve-se elaborar uma estratégia ventilatória individualizada ${ }^{6}$.

A fisioterapia motora precoce deve ser empregada com segurança em pacientes com COVID-19 durante a ECMO, mas, para tanto, deve ser realizada por equipe multidisciplinar especializada e com dimensionamento adequado ao nível de atendimento. O foco na reabilitação destes pacientes está principalmente na monitorização dos dispositivos relacionados à ECMO e ajustes necessários ao suporte circulatório e ventilatório. $\mathrm{O}$ diferencial está em garantir a segurança do paciente durante o atendimento, para tanto é essencial conhecer os dispositivos, montagem com posicionamento correto e monitorização adequada para cada etapa da reabilitação $0^{17-21}$.

Este é o posicionamento da ASSOBRAFIR em relação à indicação da ECMO e à atuação dos fisioterapeutas durante o seu uso em pacientes com COVID-19. Esperamos, com isso, contribuir para a orientação e esclarecimento dos fisioterapeutas neste momento de incertezas. A ASSOBRAFIR está atenta à evolução dos acontecimentos e sempre que identificar necessidade emitirá nova comunicação.

Atualizado em 08 de junho de 2020. 


\section{Referências}

1. Chaves RCF, Rabello Filho R, Timenetsky KT, Moreira FT, Vilanova LCS, Bravim BA, et al. Extracorporeal membrane oxygenation: a literature review. Rev Bras Ter intensive. 2019 Sep;31(3): 410-24. doi: 10.5935/0103-507x.20190063. Epub Oct 14, 2019.

2. Brogan TV, Lequier L, Lorusso R, MacLaren G, Peek G. Extracorporeal life support: the ELSO Red Book. $5^{\text {th }}$ ed. Ann Arbor, MI: Extracorporeal Life Support Organization; 2017.

3. Bartlett RH, Ogino MT, Brodie D, McMullan DM, Lorusso R, MacLaren G, et al. Initial ELSO Guidance Document: ECMO for COIVD-19 Patients with Severe Cardiopulmonary Failure. ASAIO J. 2020 May;66(5):472-474. doi: 10.1097/MAT.0000000000001173.

4. Combes A, Hajage D, Capellier G, Demoule A, Lavoué S, Guervilly C, et al. Extracorporeal Membrane Oxygenation for Severe Acute Respiratory Distress Syndrome. N Engl J Med. 2018 May 24;378(21):1965-75. doi: 10.1056/NEJMoa1800385.

5. Liang T, editor. Handbook of COVID-19 Prevention and Treatment [Internet]. Hangzhou, Zhejiang: Zhejiang University School of Medicine; [2020]. Available from: https://esge.org/documents/Handbook of COVID-19 Prevention and Treatment.pdf.

6. Spinelli E, Carlesso E, Mauri T. Extracorporeal support to achieve lung-protective and diaphragm-protective ventilation. Curr Opin Crit Care. 2020 Feb;26(1):66-72. doi: 10.1097/ MCC.0000000000000686. .

7. Gattinoni L, Tonetti T, Quintel M. How best to set the ventilator on extracorporeal membrane lung oxygenation. Curr Opin Crit Care. 2017 Feb;23(1):66-72. doi: 10.1097/MCC.0000000000000376.

8. Youngquist ST, Stoecklein H, Slack S, Tonna JE. ECMO \& ECPR. JEMS [Internet]. 2017. Available from: https://www.jems.com/2017/12/01/ecmo-ecpr/.

9. Zapol WM, Snider MT, Hill JD, Fallat RJ, Bartlett RH, Edmunds LH, et al. Extracorporeal membrane oxygenation in severe acute respiratory failure. A randomized prospective study. JAMA. 1979 Nov 16;242(20):2193-6. doi: 10.1001/jama.242.20.2193.

10. Ratliff JL, Hill JD, Fallat RJ, Parrot J, Tucker HJ. Complications Associated with Membrane Lung Support by Venoarterial Perfusion. Ann Thorac Surg. 1975 May;19(5):537-9. doi: 10.1016/s00034975(10)64429-3.

11. Luecke T, Pelosi P. Clinical review: Positive end-expiratory pressure and cardiac output. Crit Care. 2005;9(6):607-21. doi: 10.1186/cc3877. Epub 2005 Oct 18.

12. Vieillard-Baron A, Matthay M, Teboul JL, Bein T, Schultz M, Magder S, et al. Experts' opinion on management of hemodynamics in ARDS patients: focus on the effects of mechanical ventilation: focus on the effects of mechanical ventilation. Intensive Care Med. 2016 May;42(5):739-749. doi: 10.1007/s00134-016-4326-3. Epub 2016 Apr 1.

13. Franchineau G, Bréchot N, Lebreton G, Hekimian G, Nieszkowska A, Trouillet JL, et al. Bedside contribution of electrical impedance tomography to setting positive end-expiratory pressure for extracorporeal membrane oxygenation-treated patients with severe acute respiratory distress syndrome. Am J Respir Crit Care Med. 2017 Aug 15;196(4):447-457. doi: 10.1164/rccm.2016051055OC. 
14. Serpa Neto A, Deliberato RO, Johnson AEW, Bos LD, Amorim P, Pereira SM, et al. Mechanical power of ventilation is associated with mortality in critically ill patients: an analysis of patients in two observational cohorts: an analysis of patients in two observational cohorts. Intensive Care Med. 2018 Nov;44(11):1914-22. doi: 10.1007/s00134-018-5375-6. Epub 2018 Oct 5.

15. Quintel M, Busana M, Gattinoni L. Breathing and Ventilation during Extracorporeal Membrane Oxygenation: how to find the balance between rest and load: How to Find the Balance between Rest and Load. Am J Respir Crit Care Med. 2019 Oct 15;200(8):954-956. doi: 10.1164/rccm.2019061164ED.

16. Telias I, Junhasavasdikul D, Rittayamai N, Piquilloud L, Chen L, Feruson ND, et al. Airway occlusion pressure as an estimate of respiratory drive and inspiratory effort during assisted ventilation. Am J Respir Crit Care Med. 2020 May 1;201(9):1086-1098. doi: 10.1164/rccm.201907-1425OC.

17. Abrams D, Javidfar J, Farrand E, Mongero LB, Agerstrand CL, Ryan P, et al. Early mobilization of patients receiving extracorporeal membrane oxygenation: a retrospective cohort study: a retrospective cohort study. Crit Care. 2014 Feb 27;18(1):R38. doi: 10.1186/cc13746.

18. Polastri M, Loforte A, Dellamore A, Nava S. Physiotherapy for patients on awake extracorporeal membrane oxygenation: a systematic review: A systematic review. Physiother Res Int. 2016 Dec;21(4):203-209. doi: 10.1002/pri.1644. Epub 2015 Aug 14.

19. Eden A, Purkiss C, Cork G, Baddeley A, Morris K, Carey L, et al. In-patient physiotherapy for adults on veno-venous extracorporeal membrane oxygenation - United Kingdom ECMO Physiotherapy Network: a consensus agreement for best practice: A consensus agreement for best practice. J Intensive Care Soc. 2017 Aug;18(3):212-20. doi: 10.1177/1751143717705801. Epub 2017 Jun 14.

20. Ko Y, Cho YH, Park YH, Lee H, Suh GY, Yang JH, et al. Feasibility and Safety of Early Physical Therapy and Active Mobilization for Patients on Extracorporeal Membrane Oxygenation. ASAIO J. Sep-Oct 2015;61(5):564-8. doi: 10.1097/MAT.0000000000000239.

21. Martinez BP, de Andrade FMD. Estratégias de Mobilização exercícios terapêuticos precoces para pacientes em ventilação mecânica por insuficiência respiratória aguda secundária ã COVID-19. Comunicação Oficial COVID-19 ASSOBRAFIR [Internet]. São Paulo: Associação Brasileira de Fisioterapia Cardiorrespiratória e Fisioterapia em Terapia Intensiva; 2020 [cited 2020 Apr 20]. Available from: https://assobrafir.com.br/covid-19-mobilizacao-precoce-na-insuficiencia-respiratoria-aguda/.

Submissão em: 07/06/2020

Aceite em: 15/07/2020 